\title{
The Study of the Preliminary Forming Process of the Highly-Homogeneous Mixture Consisting of the Aluminum Powder - CNT in the Production Technology of Composite Materials
}

\author{
Nikolai Vetkasov $^{1, *}$, Valeriy Sapunov ${ }^{1}$, and Sergey Varlamov ${ }^{1}$ \\ ${ }^{1}$ Ulyanovsk State Technical University, Machine building faculty, 32 Severnyy Venets st, Ulyanovsk, \\ 432027, Russia
}

\begin{abstract}
The issue of the nanocomposites' synthesis using of aluminum matrixes, reinforced with carbon nanotubes, with high physical and mechanical performance capabilities, as related to achieving of the homogeneous dispersion of carbon nanotubes in the composite's aluminum matrix has been considered. Basics of the preparation technology have been developed and requirements for parameters of the so-called «normalized» mixture (highly-homogeneous in terms of volume of the dry mixture «aluminum powder - single-walled carbon nanotubes), intended for the efficient synthesis of composite granules by the mechanical alloying have been determined.
\end{abstract}

\section{Introduction}

At the present time multi-walled (MCNT) and single-walled carbon nanotubes (SCNT) are becoming more common as the reinforcing material in metallomatrix composite materials. Nanocomposites based on aluminum matrixes are of special interest. They are widely sought after in the aerospace industry and the motor-vehicle industry [1-4].

After analyzing of the results of the published papers regarding the study and the creation of aluminomatrix composite materials using of CNT we have found out that the key issue of the nanocomposites' synthesis using of aluminum matrixes, reinforced with CNT, with high physical and mechanical performance capabilities, is to achieve the homogeneous dispersion of CNT in the aluminum matrix of the composite [5-8].

In the eyes of many experts the efficient dispersion of CNT in the powder of the aluminum matrix in case of its successful realization during the synthesis of composite granules from composite powders and then of ready composites using of the intensive mechanical deformation including in particular the mechanical alloying method, is a basis for achieving of high physical and mechanical performance capabilities of composites. Authors of these papers truly state that clustering and agglomerating of CNT is a crucial technological issue [9-12].

\footnotetext{
*Corresponding author: nppwt@ulstu.ru
} 
Nonetheless, both the abovementioned and some other experts see that the whole production process of composite granules is realized under the constant deforming energy (with the same rotating speed of alloying installations (attritors, ball and planetary mills)). Authors of studies varied sizes of matrix powder fractions, the concentration of the introduced CNT, the processing time, the medium, process regulating agents, weight ratios of the mixture and balls, etc [13-17].

Authors of this paper suggest another approach to solving of the issue being considered. It resides in the fact that to receive the homogeneous dispersion of CNT in the aluminum matrix of the composite it is suggested to introduce in the synthesis technology of composite granules a special technological operation related to the mixture normalization (of the matrix powder and CNT) with the degraded energy deposition of grinding bodies on powder mixture components which exclude the appearance of the cold welding.

This paper shows the results of experimental studies related to the searching for the efficient technology of the mixture normalization during the composite granules' synthesis for aluminomatrix composites by the mechanical alloying of the aluminum powder PAD-1 using of single-walled carbon nanotubes TUBALL (SCNT).

\section{Materials}

As sources the following materials have been used:

- primary dispersed aluminum powder PAD-1 (STO22436138-006-2006) with the fraction size of up to $30 \mu \mathrm{m}$;

- single-walled carbon nanotubes TUBALL 01RW01 (TS 2166-001-91735575-2014) with the average outer diameter of not more than $3 \mathrm{~nm}$ and the full specific surface of not less than $300 \mathrm{~m}^{2} / \mathrm{g}$.

Stearic acid (GOST 6484 - 96) has been used as a surface acting agent regulating of the mixture normalization process.

\section{Research technique}

To produce composite granules, the mixture of the following formulation has been used as a basic one:

- the matrix powder PAD-1 - 97,5\% of the weight;

- single-walled carbon nanotubes TUBALL (SCNT) - $2 \%$ of the weight;

- stearic acid $-0,5 \%$ of the weight.

The mixture has been normalized and then alloyed in the mechanical reactor of the original design which includes two inside elements vibrators fastened on a single horizontal axis creating of vibration and power impacts on the wall grinding bodies in two mutually perpendicular directions with the phase shift of $30^{\circ}[9,10]$.

Morphology and size of particles have been estimated using of the digital microscope Dino-Lite AM4112PT and the optical microscope Altami MET 6T. The Rockwell hardness of specimens (HRB scale) has been measured using of the hardness measuring instrument TP150M. This very control method has been selected from the consideration that the reliable material hardness estimation is possible in case of the indentor's impact on the large volume of the material.

Cold and hot pressing has been carried out using of the press ПБ6324Б with the force of up to $1000 \mathrm{~N}$ with a special tooling designed and produced by authors and the auxiliary equipment.

Studies have been carried out in accordance with a new approach to the implementation of the synthesis technology of composite granules suggested by authors. This approach 
involves introducing in the synthesis technology of composite granules of a special technological operation of preliminary preparing of the powder mixture for the mechanical alloying.

This operation, which is called the mixture «normalization» process, provides processing of the primary mixture of the matrix aluminum powder and alloying CNT at low impact energy levels of grinding bodies on mixture components in the mechanical reactor. Energy is limited by the level when the cold welding is impossible. The uniform volume distribution of CNT in the matrix powder is realized with the production of the normalized mixture with the low bulk density. The normalized mixture consists of the compressed particles of the primary aluminum powder in a single developed surface with multiple fissures and gathers and «captured» particles of CNT agglomerates.

It shall be noted that the normalization process assumes decreasing of SCNT agglomerates to certain sizes comparable with sizes of granules of the primary powder PAD-1. The availability of agglomerates is a preservation assurance of CNT in the original state (absence of critical damages in CNT) at the mixture normalization stage. Nonetheless, the mandatory condition is to achieve their mainly bound state with the deformed particles of the matrix aluminum powder during the normalization. Most of agglomerates shall be «captured» by the developed surface of the alloyed aluminum powder. In the authors' opinion the achievement of this state under the uniform volume distribution of CNT in the aluminum powder is a necessary and sufficient condition for the uniform distribution of CNT over the volume of composite granules.

If the energy level for the mixture normalization is set correctly, its bulk density will be stabilized with a short period of time. Nonetheless, the increased duration of the normalization does not lead to changing of the morphology and the bulk density of the mixture. The normalization regime is set and it means that it is finished.

After the mixture normalization the synthesis of composite granules during the mechanical alloying is carried out under energetic impacts multiply exceeding energetic impacts during the normalization process.

The basic normalization technology of the primary mixture of PAD-1 and SCNT and its further mechanical alloying has been carried out using of the double-vibrator mechanical reactor of the abovementioned author's design with the following parameters:

- the rotating speed of the vibrator during the mixture normalization has been $200 \mathrm{r} / \mathrm{min}$ (with using of the reverse motion every $10 \mathrm{~min}$ );

- the rotating speed of the vibrator during the mechanical alloying has been $400 \mathrm{r} / \mathrm{min}$ (with using of the reverse motion every $30 \mathrm{~min}$ ). Nonetheless, during the mechanical alloying the working chamber has been periodically turned (clockwise and then anticlockwise) around the axis every $15 \mathrm{~min}$ for the angle of $90^{\circ}$;

- grinding bodies have been balls of steel ShKh15 with the diameter of $10 \mathrm{~mm}$;

- the mass ratio of balls and the mixture has been 55:1;

Processing has been carried out in the argon medium.

The duration of the mixture normalization has been varied at four levels: 40, 80, 120 and $160 \mathrm{~min}$.

The following criteria have been accepted as criteria of the sufficient duration of the mixture normalization:

- the reduction of the bulk density of the mixture up to $0,3 \ldots 0,4 \mathrm{~g} / \mathrm{cm}^{3}$ at the primary bulk density of PAD-1 equal to $1,08 \ldots 1,1 \mathrm{~g} / \mathrm{cm}^{3}$;

- the limitation of the size of CNT agglomerates which cannot exceed the maximum size of the primary alloyed matrix powder $(30 \mu \mathrm{m})$;

- the transition of the normalization process in the stabilized mode which is characterized with the absence of any changes in the bulk density of the mixture during not less than $20 \mathrm{~min}$. 
The duration of the mechanical alloying process has been $180 \mathrm{~min}$. The synthesis process has been finished when the bulk density of composite granules has achieved the values not less than the bulk density of the primary matrix powder.

During and after the normalization process the morphology of particles has been registered and the state of particles as related to the "capture» of nanotubes agglomerates has been estimated. After the mechanical alloying the morphology and the size distribution of produced composite granules have been registered.

\section{Study results}

Place the figure as close as possible after the point where it is first referenced in the text. If there is a large number of figures and tables it might be necessary to place some before their text citation.

At the first stage the series of successive independent processes related to the mixture normalization and the further mechanical alloying of produced batches of the normalized mixture have been carried out. After the normalization common factors of changes in the bulk density and the morphology of the mixture have been analyzed depending on the duration of the normalization. After the mechanical alloying of the normalized mixture common factors of changes in the bulk density, the size distribution and the morphology of composite granules have been analyzed.

The normalization process is accompanied with the reduction of the bulk density of the mixture to the set value of $0,33 \ldots 0,35 \mathrm{~g} / \mathrm{cm}^{3}$. This attests to the fact that the applied level of the low-energy impact which excludes the cold welding process characterized with forming of composite granules and increasing of their bulk density has been selected correctly.

The results of the morphological analysis of particles of the normalized mixture show that the objective is achieved. SCNT agglomerates are captured by compressed particles of the matrix aluminum. Agglomerates are captured by both the periphery and the surface of deformed aluminum particles with multiple fissures and gathers. Nonetheless, sizes of agglomerates, highly likely consisting of primary undamaged SCNT, do not exceed the primary size of the matrix PAD-1 (up to $30 \mu \mathrm{m}$ ).

During the mechanical alloying process of the normalized mixture high-volume composite granules are formed. Their size and density are increasing with simultaneous increasing of the bulk density. The bulk weight is increased to the set minimum value, achieved as the result of the mixture normalization, close to the bulk weight of the primary matrix powder PAD-1 (Fig. 1). In the series of carried out experiments the duration of the mechanical alloying has been limited with time when the bulk weight has increased up to $1,08 \ldots 1,12 \mathrm{~g} / \mathrm{cm}^{3}$.

When analyzing of the morphology of composite granules, obtained after the mechanical alloying, as related to the transformation of state of SCNT agglomerates formed as the result of the mixture normalization, their dissolution has been identified. It is highly likely connected with the absorption of their content by the massive of granules. This fact is certified with coloring (changing of color) of the surface of observed granules in the prominent dark-grey color contrasting with the silver color of the clear matrix aluminum powder during the mixture normalization and the mechanical alloying at the same regimes. Coloring certifies that SCNT are present immediately in the surface level of composite granules made of surfaces of finer granules forming its integral part. Interestingly, it is thought that this fact also relates to inner division surfaces between finer granules of which during the mechanical alloying as the result of cold welding observed composite granules are formed. On the other hand this explains the high distribution homogeneity of SCNT in masses of both composite granules and the produced composite material. 


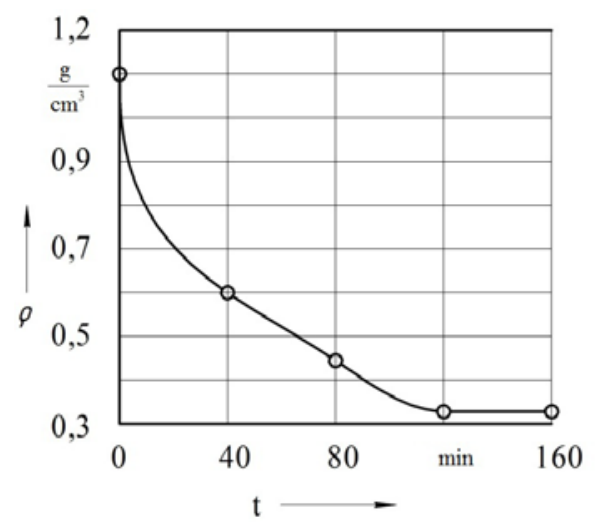

a)

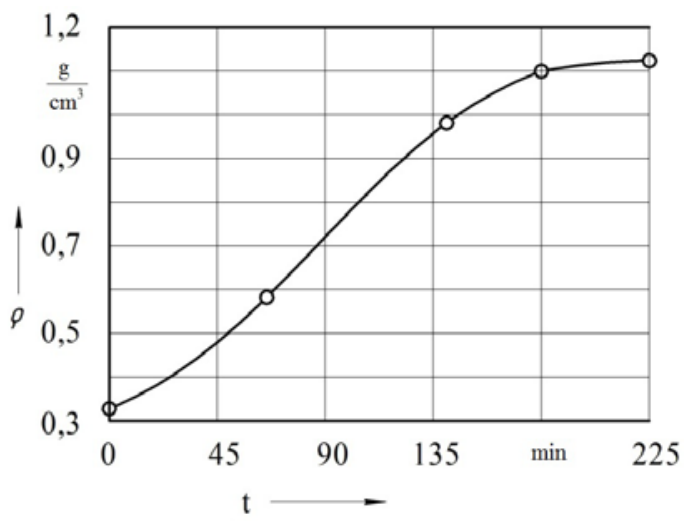

b)

Fig. 1. Dependence of the bulk density of the mixture $\rho$ on the duration of the processing $t$ in the mechanical reactor: $\mathrm{a}$ - a stage of the mixture normalization; $\mathrm{b}$ - a stage of the mechanical alloying

\section{Conclusions}

1. The technology for the mixture normalization has been developed. This technology gives an opportunity to solve the issue related to providing of the homogeneous distribution of $\mathrm{SNC}$ in the mass of the composite material at the first stage of the mixture preparation for the mechanical alloying. Essential requirements for the normalization operation and its implementation regime have been formulated.

2. It has been testified that the application of the developed technology of the mixture normalization provides the amplification of hardness performance capabilities of semifinished composite materials by not less than $25 \%$ compared to technologies without applying of the normalization.

3. Results of the paper can be used as a basis for the technological improvement of producing of aluminomatrix composites with high physical and mechanical performance capabilities.

\section{References}

1. C. Yang, Y. Zong, Z. Zheng, D. Shan, Experimental and theoretical investigation on the compressive behavior of aluminum borate whisker reinforced $2024 \mathrm{Al}$ composites, Materials Characterization, 96, pp. 84-92 (2014)

2. L. Wang, H. Choi, J.M. Myoung, W. Lee, Mechanical alloying of multi-walled carbon nanotubes and aluminium powders for the preparation of carbon/metal composites, Carbon, 47, pp. 3427-3433 (2009)

3. M. Susniak, J. Karwan-Baczewska, J. Dutkiewicz, M. Actis Grande, M. Rosso, An experimental study of aluminum alloy matrix composite reinforced sic made by hot pressing method, Archives of Metallurgy and Materials, 60, pp. 1523-1527 (2015)

4. S.S. Narayanan, G. Manickavasaham, S. Moorthy, Experimental Investigation of Aluminium alloy with MWCNT Composite to increase the echanical Properties by Stir Casting Method, IOSR Journal of Mechanical and Civil Engineering, v. 12 (4) ver. II, pp. 30-34 (2015)

5. E.P. Shalunov, M.A. Shvedov, I.V. Arkhipov, Synthesis of dispersoids in the reaction mechanical alloying of powdered aluminum with carbon, Bulletin of the Chuvash University, 2 pp. 165-172 (2014) 
6. Yu.V. Kuzmich, I.G. Kolesnikova, V.I. Serba, B.M. Freudin, Mechanical alloying (Science, Moscow, 2005)

7. S.J. Yoo, S.H. Han, W.J. Kim, Strength and strain hardening of aluminum matrix composites with randomly dispersed nanometer-length fragmented carbon nanotubes, Scr. Mater., 68, pp. 711-714 (2013)

8. N. Vetkasov, S. Ulitin, O. Krupennikov, V. Sapunov, A. Ankilov, Simulation of thermal strength of the process of flat grinding of workpieces in intermittent circles, MATEC Web of Conferences, 298, 00022 (2019)

9. N. Vetkasov, A. Kapustin, V. Sapunov, Development and Investigation of The Production Process of the Aluminum Matrix Composition Mechanically Alloyed With Single-Walled Carbon Nanotubes and the Composition Material Made of it, MATEC Web of Conferences, 297, 04001 (2019)

10. N. Vetkasov, A. Kapustin,V. Sapunov, Study of the process of mechanical doping of aluminum with nanoscale allotropic carbon modifications, Materials Today: Proceedings, v. 19(5), pp. 2299-2303 (2019)

11. V. Sapunov, N. Vetkasov, L. Khudobin, The study of the health of grinding wheels on a bakelite bunch, heat-treated in a microwave field, Materials Today: Proceedings, v. 38(4), pp. 1711-1713 (2021)

12. N. Vetkasov, L. Khudobin,V. Sapunov, S. Varlamov, Obtaining, microstructure and microhardness of carbon-doped aluminomatrix composites, Materials Today: Proceedings, v. 38(4), pp. 1706-1710 (2021)

13. L. Ci, Z. Ryu, P.N.Y. Jin, M. Rühle, Investigation of the interfacial reaction between multi-walled carbon nanotubes and aluminum, Acta. Mater., 54, pp. 5367-5375 (2006)

14. N. Nayan, S.V.S.N. Murty, S.C. Sharma, K.S. Kumar, P.P. Sinha, Calorimetric study on mechanically milled aluminum and multiwall carbon nanotube composites, Materials Characterization, 62, pp. 1087-1093 (2011)

15. D.Yu. Pushcharovsky, X-ray analysis of minerals (Geoinformmak, Moscow, 2000).

16. A.I. Gusev, Nanomaterials, nanostructures, nanotechnologies, second edition (Science - Fizmatlit, Moscow, 2007)

17. A.I. Gusev, A.S. Kurlov, Certification of nanocrystalline materials by particle size (grains), Metallophysics and the latest technology, 30, pp. 679-694 (2008) 